\title{
Diosgenin improves functional recovery from sciatic crushed nerve injury in rats
}

\author{
Byung-Ki Lee', Chang-Ju Kim², Mal-Soon Shin ${ }^{3}$, Young Sam Cho ${ }^{4, *}$ \\ 'Department of Physical Therapy, Daewon University College, Jecheon, Korea \\ ${ }^{2}$ Department of Physiology, College of Medicine, Kyung Hee University, Seoul, Korea \\ ${ }^{3}$ School of Global Sport Studies, Korea University, Sejong, Korea \\ ${ }^{4}$ Department of Urology, Kangbuk Samsung Hospital, Sungkyunkwan University School of Medicine, Seoul, Korea
}

Peripheral nerve injuries are commonly encountered clinical problem and often result in chronic pain and severe functional deficit. Diosgenin is a plant steroidal saponin and has anti-inflammatory and anticancer effects. In the present study, we investigated the effect of diosgenin on functional recovery following sciatic crushed nerve injury in rats. Walking track analysis for the functional recovery which can be quantified with the sciatic function index (SFI) was conducted. Immunohistochemistry for c-Fos in the ventrolateral periaqueductal gray (vIPAG) and paraventricular nucleus (PVN) and western blot for brain-derived neurotrophic factor (BDNF), tyrosine kinase B (TrkB), cyclooxygenase-2 (COX-2), and inducible nitric oxide synthesis (iNOS) in the sciatic nerve were performed. The right sciatic nerve was crushed for $30 \mathrm{sec}$ using a surgical clip. The animals in the diosgenin-treated groups received orally once a day at the respective doses for 7 consecutive days, starting one day after surgery. Sciatic crushed nerve injury showed characteristic gait changes showing decrease of SFI value. Diosgenin treatment increased the SFI value and suppressed nerve injury-induced c-Fos expression in the vIPAG and PVN. Diosgenin treatment inhibited nerve injury-induced increase of BDNF, TrkB, COX-2, and iNOS expressions. It is possible that diosgenin can be used as the new therapeutic agent for pain control and functional recovery following peripheral nerve injury.

Keywords: Sciatic nerve injury, Functional recovery, Diosgenin, c-Fos, Brained-derived neurotrophic factor

\section{INTRODUCTION}

Peripheral nerve injuries are commonly encountered clinical problem and often result in chronic pain and severe functional deficit. The affected limb displays characteristics of painful neuropathy, such as hyperalgesia, pain related gait, and swelling. Ascending spinal pathways subsequently distribute nociceptive information to multiple cortical, limbic structures and hypothalamus involved in the pain (Hunt and Mantyh, 2001). Transmission of nociceptive information may be altered by many neuronal circuits within the central nervous system. The periaquaductal gray (PAG) and paraventricular nucleus (PVN) are an important sites in ascending pain transmission.

$c$-Fos, an immediate early gene, is used as a marker for stimuliinduced metabolic changes of neurons, and its expression is also induced under various conditions (de Medeiros et al., 2003). c-Fos expression is recognized as a marker of increased neuronal activity (Lee et al., 2003). Sciatic nerve ligation increased c-Fos expression in the frontal cortex, thalamus, and PAG in rats (Narita et al., 2003).

The brain-derived neurotrophic factor (BDNF) is an important neuromodulator of synaptic plasticity in the peripheral nerve injury, and it is a member of the neurotrophic family that promotes the survival of specific neurons during development (Woolf and Costigan, 1999). It has higher affinity for the receptor tyrosine kinase B (TrkB) (Maness et al., 1994).

Prostaglandins (PGs) are key inflammatory mediators that are converted from arachidonic acid by cyclooxygenase (COX). There are two isoforms of COX: cyclooxygenase-1 (COX-1) is constitutively expressed in nearly all tissue and provides PGs to maintain physiological functions, such as cytoprotection of the stomach and
${ }^{*}$ Corresponding author: Young Sam Cho (iD https://orcid.org/0000-0002-2966-7971 Department of Urology, Kangbuk Samsung Hospital, Sungkyunkwan University School of Medicine, 29 Saemunan-ro, Jongno-gu, Seoul 03181, Korea Tel: +82-2-2001-2237, Fax: +82-2-2001-2247, E-mail: choys1011@naver.com Received: June 2, 2018 / Accepted: July 21, 2018
This is an Open Access article distributed under the terms of the Creative Commons Attribution Non-Commercial License (http://creativecommons.org/licenses/by-nc/4.0/) which permits unrestricted non-commercial use, distribution, and reproduction in any medium, provided the original work is properly cited. 
regulation renal blood flow (Vane et al., 1998). COX-2 plays a major role in the biosynthesis of PGs from arachidonic acid, and COX-2 is up-regulated in the injured nerve by partial sciatic nerve ligation (Ma and Eisenach, 2002, 2003).

Nitric oxide (NO) is a messenger and effecter molecule in a variety of tissues (Lowenstein and Greenberg, 1996). NO was identified as a neurotransmitter, and it acts as a potent vasodilator in physiological condition (Baek et al., 2016). The production of $\mathrm{NO}$ is regulation by intracellular nitric oxide synthases (NOS), and three types of NOS have been identified: endothelial nitric oxide synthase (eNOS), neural nitric oxide synthase (nNOS), and inducible nitric oxide synthase (iNOS). Of these, iNOS is implicated in the pathophysiology of inflammatory and neuropathic pain (De Alba et al., 2006). iNOS expressed by glia is involved in the mechanisms of hyperalgesia.

Diosgenin is a steroidal saponin, which is found in several plant species, and particularly in fenugreek or wild yam (Dioscorea spp.) roots. Plant saponin has anti-inflammatory, anti-oxidative, and anticancer effects (Raju et al., 2004). Au et al. (2004) reported that diosgenin is structurally similar to progesterone and estrogen, and diosgenin caused acute endothelium-independent coronary artery relaxation. Saponin-rich plant acts as an anti-inflammatory agent through inhibiting COX-2 and iNOS expressions, thereby inhibiting production of PGs (Li et al., 2006). Moharram and El-Shenawy (2007) and Wang et al. (2008) also reported that plant saponin exhibited anti-nociceptive action.

The sciatic function index (SFI) compares parameters from the normal and experimental footprints by a mathematical formula, and provides information concerning the recovery of sensory-motor connections and cortical integration related to gait function mediated by the sciatic nerve (Byun et al., 2005).

In the present study, we investigated the effect of diosgenin on sciatic crushed nerve injury in rats. For this, walking track analysis for the functional recovery which can be quantified with the SFI was conducted. Immunohistochemistry for the c-Fos expression in the ventral lateral PAG (vlPAG) and PVN and western blot analysis for the expressions of BDNF, TrkB, COX-2, and iNOS in the sciatic nerve were conducted.

\section{MATERIALS AND METHODS}

\section{Animals and treatments}

Male Sprague-Dawley rat weighing 350 $\pm 10 \mathrm{~g}$ (12 weeks of age) were used. The experimental procedures were performed in accordance with the animal care guideline of National Institutes of Health and the Korea Academy of Medical Sciences. The rats were randomly divided into five groups ( $\mathrm{n}=10$ in each group): the shame operation group, the sciatic crushed nerve injury group, the sciatic crushed nerve injury and $25 \mathrm{mg} / \mathrm{kg}$ diosgenin-treated group, the sciatic crushed nerve injury and $50 \mathrm{mg} / \mathrm{kg}$ diosgenin-treated group, and the sciatic crushed nerve injury and 100 $\mathrm{mg} / \mathrm{kg}$ diosgenin-treated group. The rats in the diosgenin-treated groups received diosgenin (Wako Pure Chemical Industries Ltd., Osaka, Japan) orally once a day for 7 consecutive days, starting one day after surgery.

\section{Surgical procedure}

To induce crush injury on the sciatic nerve, the previously described surgical procedure was performed (Byun et al., 2005). Right sciatic nerve was exposed by incision on the gluteal muscle under anesthesia using Zoletil $50(50 \mathrm{mg} / \mathrm{kg}$; Virbac Laboratories, Carros, France). The sciatic nerve was carefully exposed and crushed for $30 \mathrm{sec}$ using a surgical clip (pressure: $125 \mathrm{~g}$; Fine Science Tool Inc., San Francisco, CA, USA). The crushed location was between the sciatic notch and the point of trifurcation.

\section{Walking tract analysis}

Functional recovery rate after nerve injury was analyzed using a walking tract assessment, as the previously described method (Byun et al., 2005). From the footprints the following parameter were calculated: distance from the heel to the top of the $3 \mathrm{rd}$ toe (print length, PL), distance between the 1st to the 5 th toe (toe spread, TS), and distance from the 2nd to the 4th toe (intermediary toe spread, IT). These parameters were taken both from the intact left (non-operated) foot (NPL, NTS, and NIT) and from the injured right (experimental) foot (EPL, ETS, and EIT). SFI values were obtained using following equation: $\mathrm{SFI}=(-38.3 \pm \mathrm{PLF})+$ $(109.5 \pm T S F)+(13.3 \pm$ ITF $)-8.8$. Print length factor $(T S F)=($ ETS NPL)/NPL. Toe spread factor $($ TSF $)=($ EST-NTS $) /$ NTS. Intermediary toe spread factor $($ ITF $)=($ EIT - NIT $) /$ NIT. Interpolating identical values of PL, TS, and IT from the right and left hind feet are close to zero in normal rats. The SFI value of -100 indicates complete impairment.

\section{Tissue preparation}

The animals were sacrificed on 7 days after treatment. The animals were anesthetized using Zoletil $50(10 \mathrm{mg} / \mathrm{kg}$, intraperitoneally; Virbac Laboratories), transcardially perfused with $50 \mathrm{mM}$ phosphate-buffered saline, and fixed with a freshly prepared solution consisting of $4 \%$ parafomaldehyde in $100 \mathrm{Mm}$ phosphate 
buffer $(\mathrm{pH}, 7.4)$. The brains were dissected and postfixed in the same fixative overnight and transferred into a $30 \%$ sucrose solution for cryoprotection. Coronal section of $40-\mu \mathrm{m}$ thickness was made with a freezing microtome (Leica, Nussloch, Germany).

\section{c-Fos immunohistochemistry}

c-Fos immunohistochemistry was performed as the previously described method (Han et al., 2017). Free-floating tissue sections were incubated overnight with rabbit anti-c-Fos antibody (1:1,000; Santa Cruz Biotechnology, Santa Cruz, CA, USA), and the section were then incubated for $1 \mathrm{hr}$ with biotinylated anti-rabbit secondary antibody (1:200; Vector Laboratories, Burlingame, CA, USA). The sections were subsequently incubated with avidin-biotin-peroxidase complex (1:100; Vector Laboratories) for $1 \mathrm{hr}$ at room temperature. Immunoreactivity was visualized by incubating the sections in a solution consisting of $0.05 \% 3,3$-diaminobenzidine and $0.01 \% \mathrm{H}_{2} \mathrm{O}_{2}$ in $50 \mathrm{mM}$ Tris-buffer (pH, 7.6) for approximately $3 \mathrm{~min}$. The slides were air-dried over night at room temperature, and coverslips were mounted using Permount (Fisher Scientific, Pittsburgh, PA, USA).

\section{Western blot analysis}

Western blot analysis for BDNF, TrkB, COX-2, iNOS were performed according to the previous method (Baek et al., 2016; Park et al., 2017). Sciatic nerve tissue samples were homogenized in the lysis buffer and $200 \mu \mathrm{g}$ the samples were centrifuged at $14,000 \mathrm{rpm}$ at $20 \mathrm{~min}$ and supernatant collected for Western blot. Protein concentration in the sample was assayed using Bradford reagent (Bio-Rad Laboratories Inc., Hercules, CA, USA). Protein of $35 \mu \mathrm{g}$ was separated sodium dodecyl sulfate-polyacrylamide gels and transferred to nitrocellulose membrane (Whatman GmbH, Dassel, Germany). Rabbit BDNF antibody (1:1,000; Santa Cruz Biotechnology), rabbit TrkB antibody (1:1,000; Santa Cruz Biotechnology), goat COX-2 antibody (1:1,000; Santa Cruz Biotechnology), and rabbit iNOS antibody (1:1,000; Santa Cruz Biotechnology) were used as the primary antibodies. Horseradish peroxidase-conjugated anti-rabbit antibody (1:2,000; Vector Laboratories) for BDNF, TrkB, iNOS, and anti-goat antibody (1:2,000; Santa Cruz Biotechnology) for COX-2 were used as the secondary antibodies. Band detection was performed using an enhanced chemiluminescence detection system (Santa Cruz Biotechnology).
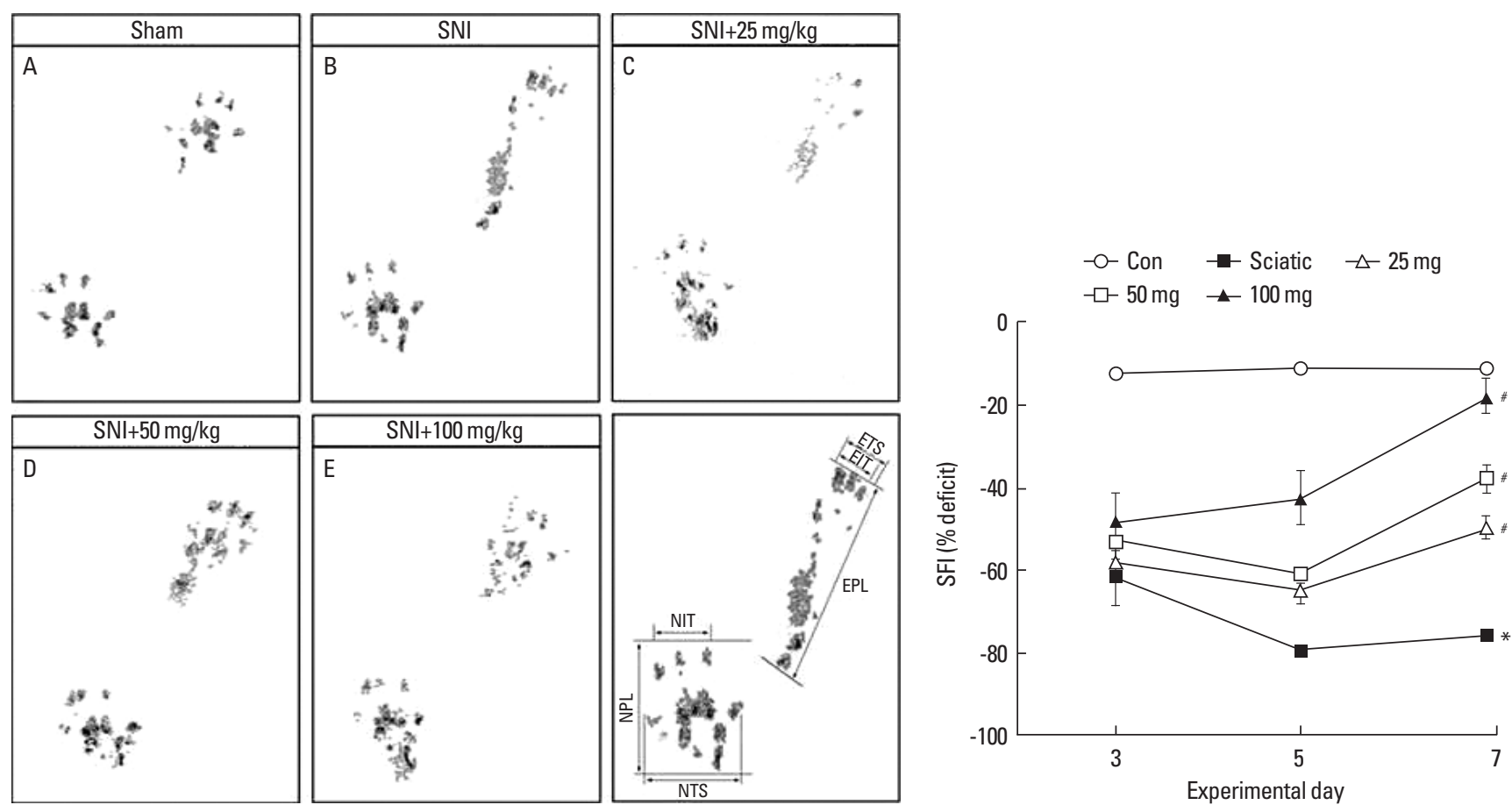

Fig. 1. Effect of diosgenin on sciatic functional index (SFI). Upper panel: walking tract footprints. A, sham operation group; B, sciatic crushed nerve injury group; C, sciatic crushed nerve injury and $25 \mathrm{mg} / \mathrm{kg}$ diosgenin-treated group; D, sciatic crushed nerve injury and $50 \mathrm{mg} / \mathrm{kg}$ diosgenin-treated group; $E$, sciatic crushed nerve injury and $100 \mathrm{mg} / \mathrm{kg}$ diosgenin-treated group. Lower panel: data of SFI. o, sham operation group; $\mathbf{a}$, sciatic crushed nerve injury group; $\Delta$, sciatic crushed nerve injury and $25 \mathrm{mg} / \mathrm{kg}$ diosgenin-treated group; $\square$, sciatic crushed nerve injury and $50 \mathrm{mg} / \mathrm{kg}$ diosgenin-treated group; $\mathbf{\Delta}$, sciatic crushed nerve injury and $100 \mathrm{mg} / \mathrm{kg}$ diosgenin-treated group. ${ }^{*} P<0.05$ compared to the sham-operation group. ${ }^{*} P<0.05$ compared to the sciatic nerve injury group. 


\section{Data analyses}

Data were expressed as the mean \pm standard error of the mean. For comparisons among the groups, one-way analysis of variance and Duncan post hoc test were performed with $P<0.05$ as indication of statistical significance.

\section{RESULTS}

\section{SFI following sciatic crushed nerve injury}

The mean SFI for each group was calculated on the 3rd, 5th, and 7 th day after sciatic crushed nerve injury. The present results indicated that diosgenin treatment promoted functional locomotor recovery following sciatic crushed nerve injury (Fig. 1).
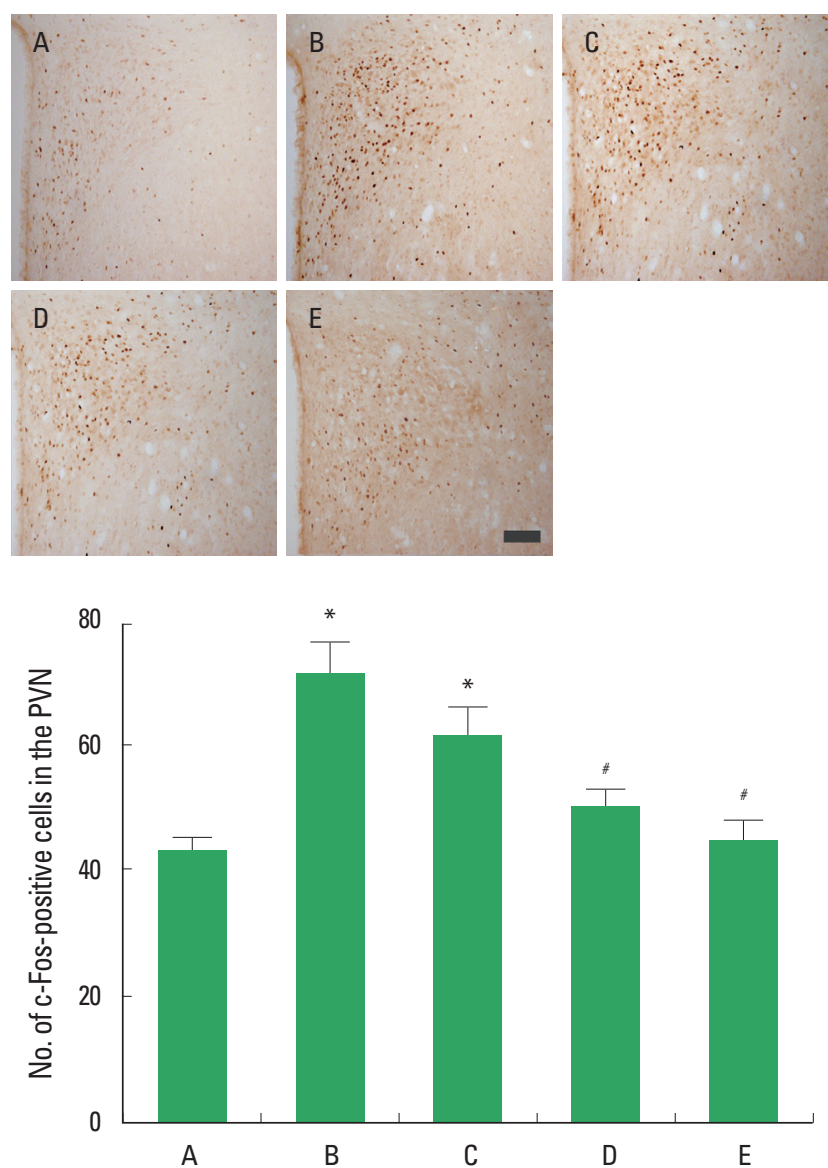

Fig. 2. Effect of diosgenin on c-Fos expression in the paraventricular nucleus (PVN). Upper panel: photomicrographs of the c-Fos-positive cells. The scale bar represents $100 \mathrm{~mm}$. Lower panel: number of c-Fos-positive cells. A, sham operation group; $B$, sciatic crushed nerve injury group; $C$, sciatic crushed nerve injury and $25-\mathrm{mg} / \mathrm{kg}$ diosgenin-treated group; $\mathrm{D}$, sciatic crushed nerve injury and 50-mg/kg diosgenin-treated group; E, sciatic crushed nerve injury and 100-mg/ kg diosgenin-treated group. ${ }^{*} P<0.05$ compared to the sham-operation group. ${ }^{\#} P<0.05$ compared to the sciatic nerve injury group.

\section{c-Fos expression in the vIPAG following sciatic crushed nerve injury}

The present results showed that sciatic crushed nerve injury enhanced c-Fos expression in the vIPAG and diosgenin treatment decreased c-Fos expression in the vlPAG (Fig. 2).

\section{c-Fos expression in the PVN following sciatic crushed nerve injury}

The present results showed that sciatic crushed nerve injury decreased c-Fos expression in the PVN and diosgenin treatment enhanced c-Fos expression in the PVN (Fig. 3).
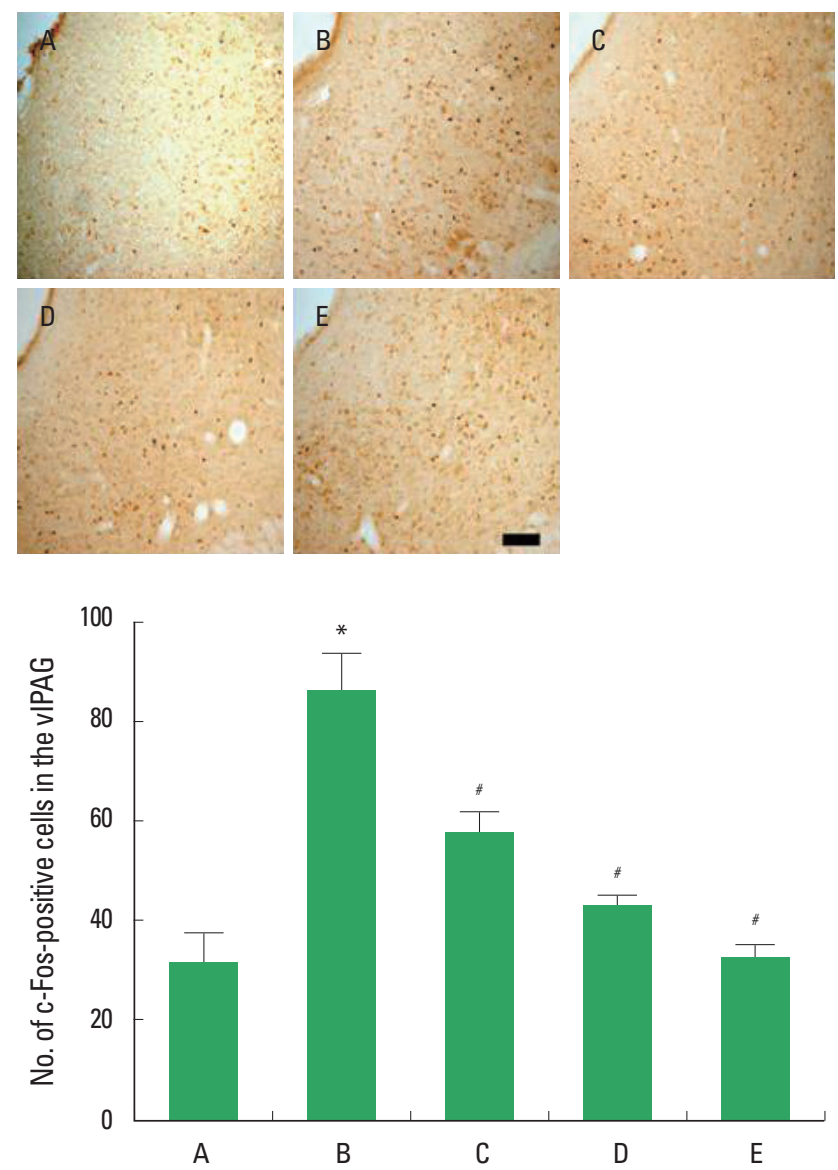

Fig. 3. Effect of diosgenin on c-Fos expression in the ventral lateral periaquaductal gray (vIPAG). Upper panel: photomicrographs of the c-Fos-positive cells. The scale bar represents $100 \mathrm{~mm}$. Lower panel: number of c-Fos-positive cells. $A$, sham operation group; $B$, sciatic crushed nerve injury group; $C$, sciatic crushed nerve injury and 25-mg/kg diosgenin-treated group; $D$, sciatic crushed nerve injury and $50-\mathrm{mg} / \mathrm{kg}$ diosgenin-treated group; $\mathrm{E}$, sciatic crushed nerve injury and $100-\mathrm{mg} / \mathrm{kg}$ diosgenin-treated group. ${ }^{*} P<0.05$ compared to the sham-operation group. ${ }^{*} P<0.05$ compared to the sciatic nerve injury group. 


\section{BDNF and TrkB expressions in the sciatic nerve following sciatic crushed nerve injury}

The present results showed that sciatic crushed nerve injury increased the levels of BDNF and TrkB, while treatment with diosgenin decreased the levels of BDNF and TrkB (Fig. 4).

\section{COX-2 and iNOS expressions in the sciatic nerve following sciatic crushed nerve injury}

The present results showed that decreased the levels of COX-2
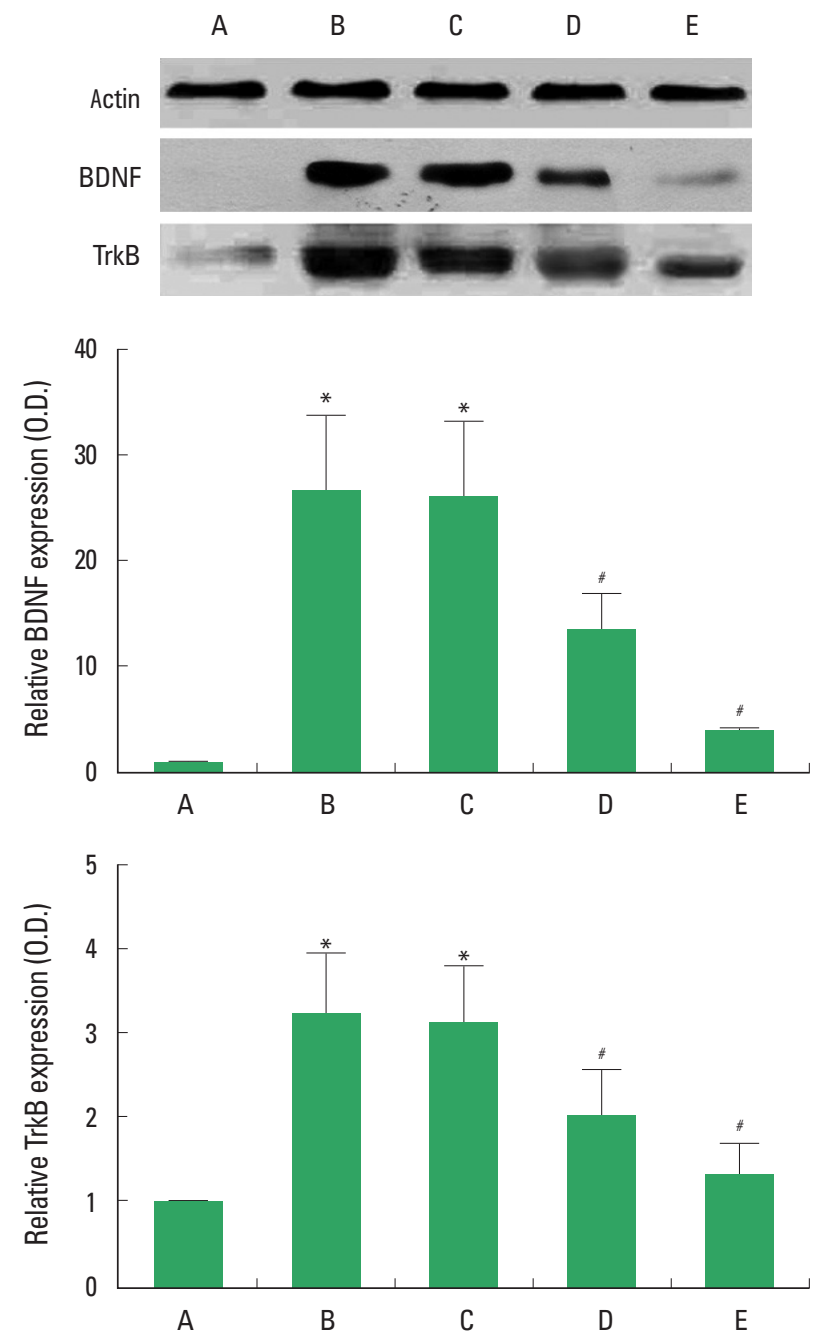

Fig. 4. Effect of diosgenin on brain-derived neurotrophic factor (BDNF) and tyrosine kinase B (TrkB) expressions in the sciatic nerve. Upper panel: representative expressions of BDNF, TrkB, and $\beta$-actin. Lower panle: relative expressions of BDNF and TrKB. A, sham operation group; $B$, sciatic crushed nerve injury group; $C$, sciatic crushed nerve injury and $25-\mathrm{mg} / \mathrm{kg}$ diosgenin-treated group; $D$, sciatic crushed nerve injury and $50-\mathrm{mg} / \mathrm{kg}$ diosgenin-treated group; $E$, sciatic crushed nerve injury and $100-\mathrm{mg} / \mathrm{kg}$ diosgenin-treated group. ${ }^{*} P<0.05$ compared to the sham-operation group. ${ }^{*} P<0.05$ compared to the sciatic nerve injury group. and iNOS, while treatment with diosgenin decreased the levels of COX-2 and iNOS (Fig. 5).

\section{DISCUSSION}

The SFI is widely used to evaluate functional gait assessment after peripheral nerve lesion (Byun et al., 2005). In the present study, sciatic nerve crushed injury showed decrease of the SFI value. However treatment with diosgenin increased SFI value, in-
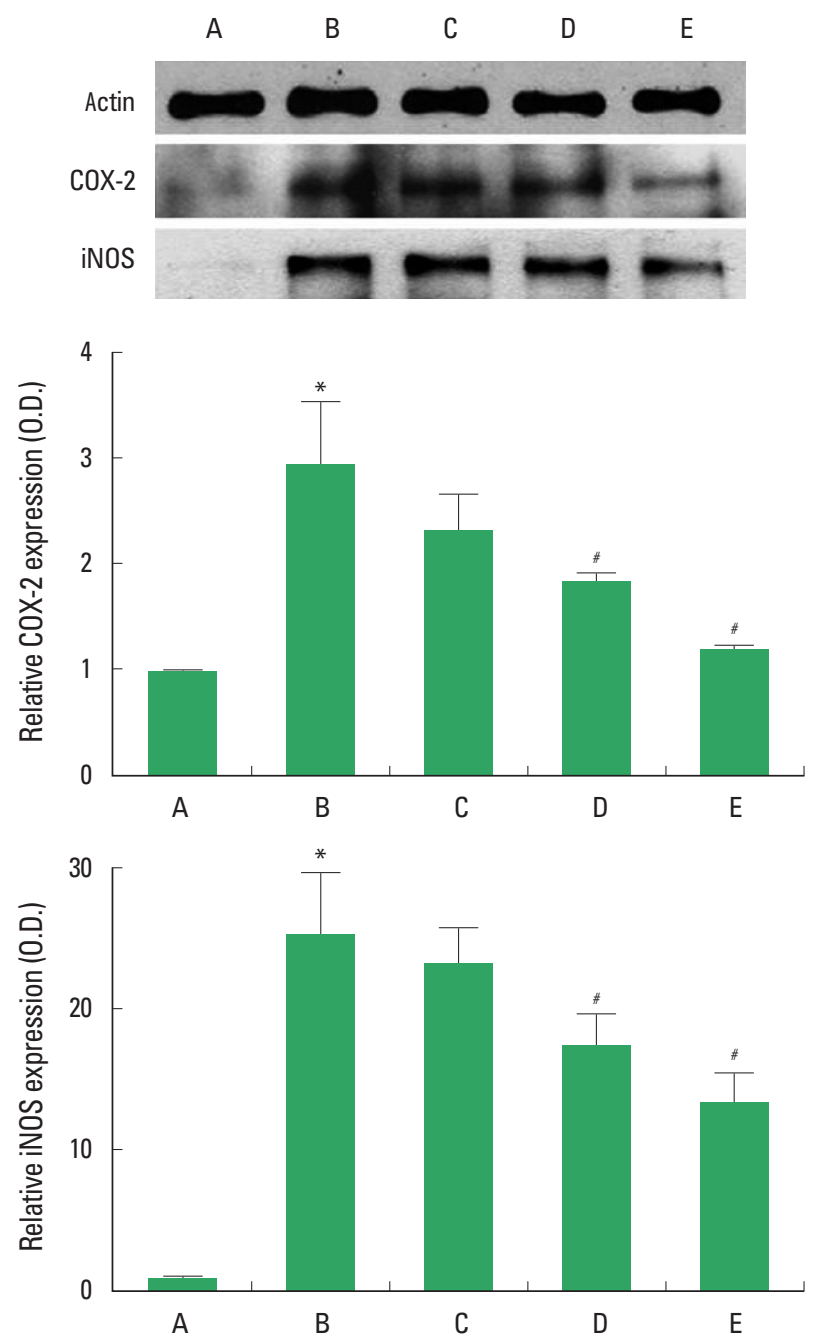

Fig. 5. Effect of diosgenin on cyclooxygenase-2 (COX-2) and inducible nitric oxide synthase (iNOS) expressions in the sciatic nerve. Upper panel: representative expressions of COX-2, iNOS, and $\beta$-actin. Lower panel: relative expressions of COX-2 and iNOS. A, sham operation group; $B$, sciatic crushed nerve injury group; $\mathrm{C}$, sciatic crushed nerve injury and $25-\mathrm{mg} / \mathrm{kg}$ diosgenin-treated group; D, sciatic crushed nerve injury and 50-mg/kg diosgenin-treated group; $E$, sciatic crushed nerve injury and $100-\mathrm{mg} / \mathrm{kg}$ diosgenin-treated group. ${ }^{*} P<0.05$ compared to the sham-operation group. ${ }^{\sharp} P<0.05$ compared to the sciatic nerve injury group. 
dicting that diosgenin increased functional recovery following sciatic crushed nerve injury.

c-Fos is a sensitive maker of the neuronal activation following sciatic nerve ligation (Nishimori et al., 2002). c-Fos expression is rapidly and transiently induced in the brain areas in response to nerve stimulation (Han et al., 2017). In the present study, c-Fos expression in the vlPAG and PVN was increased following sciatic crushed nerve injury, while diosgenin treatment suppressed the nerve injury-induce c-Fos expression in the vIPAG and PVN. The present results indicate that neurons in the vlPAG and PVN were activated by sciatic crushed nerve injury. In contrast, diosgenin suppressed noxious stimulation on sciatic nerve, resulting in decrease of neuronal activation in the vlPAG and PVN.

Neuronal BDNF expression was rapidly and transiently increased in response to peripheral nerve injury (Cho et al., 1997). Woolf and Costigan (1999) reported that BDNF may play a critical role in synaptic plasticity in nociceptive signaling. Overexpression of BDNF by sciatic nerve injury restrained recovery of locomotor function and treadmill exercise promoted functional recovery rate via suppression on BDNF mRNA expression (Byun et al., 2005). In the present study, BDNF expression in the sciatic nerve was increased by sciatic crushed nerve injury, suggesting that injury to the sciatic nerve induced BDNF expression for its regeneration. In contrast, diosgenin treatment suppressed the nerve injury-induced BDNF expression, suggesting that diosgenin facilitated regeneration of injury to the sciatic nerve, thus overexpression of BDNF was suppressed.

COX-2 causes $\mathrm{PGE}_{2}$ release in response to surgical trauma, and COX-2 is implicated in inflammation and pain (Baba et al., 2001). Peripheral inflammation up-regulates COX-2 expression in spinal cord with subsequent increase in $\mathrm{PGE}_{2}$ level, and results in development of allodynia and hyperalgesia (Guay et al., 2004). Alba et al. (2006) demonstrated that iNOS expression was overexpressed under pain conditions, and iNOS inhibitors exerted anti-inflammatory effect. Therefore, expressions of COX-2 and iNOS are closely associated with the pathogenesis of inflammatory and neuropathic pain (Baek et al., 2016). In the present study, COX-2 and iNOS expressions were increased by sciatic crushed nerve injury, indicating that injury to the sciatic nerve induced inflammation. In contrast, treatment with diosgenin inhibited COX-2 and iNOS expressions in sciatic nerve injury, representing that diosgenin exerted anti-inflammatory effect on sciatic crushed nerve injury.

Diosgenin has a large variety of biological functions, such as anti-oxidative, anticancer and anti-inflammatory effects (Moalic et al., 2001; Shishodia and Aggarwal, 2006). In the present study, diosgenin facilitated functional recovery from sciatic crushed nerve injury, thus showed suppression on BDNF and its receptor TrkB, resulted in decrease of neuronal activity in the vIPAG and PVN. Based on the present result, diosgenin can be used as the new therapeutic agent for pain control and functional recovery following peripheral nerve injury.

\section{CONFLICT OF INTEREST}

No potential conflict of interest relevant to this article was reported.

\section{REFERENCES}

Alba E, Llombart A, Ribelles N, Ramos M, Fernández R, Mayordomo JI, Tusquets I, Gil M, Barnadas A, Carabante F, Ruiz M, Vera R, Palomero I, Soriano V, González J, Colomer R. Serum endostatin and bFGF as predictive factors in advanced breast cancer patients treated with letrozole. Clin Transl Oncol 2006;8:193-199.

Au AL, Kwok CC, Lee AT, Kwan YW, Lee MM, Zhang RZ, Ngai SM, Lee $\mathrm{SM}, \mathrm{He}$ GW, Fung KP. Activation of iberiotoxin-sensitive, $\mathrm{Ca}^{2+}$-activated $\mathrm{K}^{+}$channels of porcine isolated left anterior descending coronary artery by diosgenin. Eur J Pharmacol 2004;502:123-133.

Baba H, Kohno T, Moore KA, Woolf CJ. Direct activation of rat spinal dorsal horn neurons by prostaglandin E2. J Neurosci 2001;21:1750-1756.

Baek SB, Shin MS, Han JH, Moon SW, Chang B, Jeon JW, Yi JW, Chung JY. Rocuronium bromide inhibits inflammation and pain by suppressing nitric oxide production and enhancing prostaglandin $\mathrm{E}_{2}$ synthesis in endothelial cells. Int Neurourol J 2016;20:296-303.

Byun YH, Lee MH, Kim SS, Kim H, Chang HK, Lee TH, Jang MH, Shin MC, Shin MS, Kim CJ. Treadmill running promotes functional recovery and decreases brain-derived neurotrophic factor mRNA expression following sciatic crushed nerve injury in rats. J Sports Med Phys Fitness 2005;45:222-228.

Cho HJ, Kim SY, Park MJ, Kim DS, Kim JK, Chu MY. Expression of mRNA for brain-derived neurotrophic factor in the dorsal root ganglion following peripheral inflammation. Brain Res 1997;749:358-362.

De Alba J, Clayton NM, Collins SD, Colthup P, Chessell I, Knowles RG. GW274150, a novel and highly selective inhibitor of the inducible isoform of nitric oxide synthase (iNOS), shows analgesic effects in rat models of inflammatory and neuropathic pain. Pain 2006;120:170-181.

de Medeiros MA, Canteras NS, Suchecki D, Mello LE. Analgesia and c-Fos expression in the periaqueductal gray induced by electroacupuncture at the Zusanli point in rats. Brain Res 2003;973:196-204. 
Guay J, Bateman K, Gordon R, Mancini J, Riendeau D. Carrageenan-induced paw edema in rat elicits a predominant prostaglandin $\mathrm{E}_{2}\left(\mathrm{PGE}_{2}\right)$ response in the central nervous system associated with the induction of microsomal PGE2 synthase-1. J Biol Chem 2004;279:24866-24872.

Han JH, Kim SE, Ko IG, Kim J, Kim KH. Afferent pathway-mediated effect of $\alpha 1$ adrenergic antagonist, tamsulosin, on the neurogenic bladder after spinal cord injury. Int Neurourol J 2017;21:178-188.

Hunt SP, Mantyh PW. The molecular dynamics of pain control. Nat Rev Neurosci 2001;2:83-91.

Lee MH, Kim H, Lim BV, Chang HK, Lee TH, Jang MH, Shin MC, Lee J, Shin MS, Kim CJ. Naloxone potentiates treadmill running-induced increase in c-Fos expression in rat hippocampus. Life Sci 2003;73:31393147.

Li TZ, Zhang WD, Yang GJ, Liu WY, Chen HS, Shen YH. Saponins from Polygala japonica and their effects on a forced swimming test in mice. J Nat Prod 2006;69:591-594.

Lowenstein E, Greenberg D. Next-generation informatics. A new picture of quality medicine. Physician Exec 1996;22:14-16.

Ma W, Eisenach JC. Cyclooxygenase 2 in infiltrating inflammatory cells in injured nerve is universally up-regulated following various types of peripheral nerve injury. Neuroscience 2003;121:691-704.

Ma W, Eisenach JC. Morphological and pharmacological evidence for the role of peripheral prostaglandins in the pathogenesis of neuropathic pain. Eur J Neurosci 2002;15:1037-1047.

Maness LM, Kastin AJ, Weber JT, Banks WA, Beckman BS, Zadina JE. The neurotrophins and their receptors: structure, function, and neuropathology. Neurosci Biobehav Rev 1994;18:143-159.

Moalic S, Liagre B, Corbière C, Bianchi A, Dauça M, Bordji K, Beneytout JL. A plant steroid, diosgenin, induces apoptosis, cell cycle arrest and COX activity in osteosarcoma cells. FEBS Lett 2001;506:225-230.

Moharram FA, El-Shenawy SM. Antinociceptive and anti-inflammatory steroidal saponins from Dracaena ombet. Planta Med 2007;73:11011106.

Narita M, Ozaki S, Narita M, Ise Y, Yajima Y, Suzuki T. Change in the expression of c-fos in the rat brain following sciatic nerve ligation. Neurosci Lett 2003;352:231-233.

Nishimori T, Ikeda T, Terayama R, Ishida Y, Nakamura T, Otahara N. Effect of ionotropic glutamate receptor antagonists on Fos-like immunoreactivity in the dorsal horn following transection of the rat sciatic nerve. Brain Res 2002;934:81-86.

Park JH, Ko IG, Kim SE, Jin JJ, Hwang L, Kim CJ, Yoon SH, Hong J, Chung JY, Lee DW. dexmedetomidine oral mucosa patch for sedation suppresses apoptosis in hippocampus of normal rats. Int Neurourol J 2017; 21(Suppl 1):S39-47.

Raju J, Patlolla JM, Swamy MV, Rao CV. Diosgenin, a steroid saponin of Trigonella foenum graecum (Fenugreek), inhibits azoxymethane-induced aberrant crypt foci formation in F344 rats and induces apoptosis in HT-29 human colon cancer cells. Cancer Epidemiol Biomarkers Prev 2004;13:1392-1398.

Shishodia S, Aggarwal BB. Diosgenin inhibits osteoclastogenesis, invasion, and proliferation through the downregulation of Akt, IkB kinase activation and NF-kB-regulated gene expression. Oncogene 2006;25: 1463-1473.

Vane JR, Bakhle YS, Botting RM. Cyclooxygenases 1 and 2. Annu Rev Pharmacol Toxicol 1998;38:97-120.

Wang JR, Zhou H, Jiang ZH, Wong YF, Liu L. In vivo anti-inflammatory and analgesic activities of a purified saponin fraction derived from the root of Ilex pubescens. Biol Pharm Bull 2008;31:643-650.

Woolf CJ, Costigan M. Transcriptional and posttranslational plasticity and the generation of inflammatory pain. Proc Natl Acad Sci U S A 1999;96:7723-7730. 